\title{
All-atom molecular dynamics study of hepatitis B virus containing pregenome RNA in solution
}

\author{
Kazushi Fujimoto, ${ }^{1}$ Youhei Yamaguchi, ${ }^{1}$ Ryo Urano, ${ }^{1}$ Wataru Shinoda, ${ }^{1}$ Tetsuya Ishikawa, ${ }^{2}$ \\ Katsumi Omagari, ${ }^{3}$ Yasuhito Tanaka, ${ }^{4,5}$ Atsushi Nakagawa, ${ }^{6}$ and Susumu Okazaki ${ }^{7,}$ a) \\ ${ }^{1}$ Department of Materials Chemistry, Nagoya University \\ ${ }^{2}$ Department of Integrated Health Sciences, Nagoya University \\ ${ }^{3}$ Curreio, Inc. \\ ${ }^{4}$ Department of Virology and Liver Unit, Nagoya City University \\ ${ }^{5}$ Department of Gastroenterology and Hepatology, Kumamoto University \\ ${ }^{6}$ Institute for Protein Research, Osaka University \\ ${ }^{7}$ Department of Advanced Materials Science, The University of Tokyo
}

a) Author to whom correspondence should be address

Electronic mail: okazaki@edu.k.u-tokyo.ac.jp

\begin{abstract}
Immature hepatitis B virus (HBV) captures nucleotides in its capsid for reverse transcription. The nucleotides and nucleotide analogue drugs, which are triphosphorylated and negatively charged in the cell, approach the capsid via diffusion and are absorbed into it. In this study, we performed a long-time molecular dynamics (MD) calculation of the entire HBV capsid containing pregenome RNA to investigate the interactions between the capsid and negatively charged substances. Electric field analysis demonstrated that negatively charged substances can approach the HBV capsid by thermal motion, avoiding spikes. The substances then migrate all over the floor of the HBV capsid. Finally, they find pores through which they can pass through the HBV capsid shell. Free energy profiles were calculated along these pores for small ions to understand their permeability through the pores. Anions $\left(\mathrm{Cl}^{-}\right)$showed higher free energy barriers than cations $\left(\mathrm{Na}^{+}\right.$and $\left.\mathrm{K}^{+}\right)$through all pores, and the permeation rate of $\mathrm{Cl}^{-}$was eight times slower than that of $\mathrm{K}^{+}$or $\mathrm{Na}^{+}$. Furthermore, the ions were more stable in the capsid than in the bulk water. Thus, the
\end{abstract}


HBV capsid exerts ion selectivity for uptake and provides an environment for ions, such as nucleotides and nucleotide analogue drugs, to be stabilized within the capsid.

\section{INTRODUCTION}

According to a report by the World Health Organisation (WHO), 257 million people were infected with hepatitis B virus (HBV) in 2015, among which 887,000 were killed as a result of this disease. ${ }^{1} \mathrm{HBV}$ consists of a spherical protein capsid inside an envelope that contains DNA. After the virus enters a host cell, it copies the pregenomic RNA (pgRNA) from the DNA. An immature HBV is then formed, containing the pgRNA in the capsid copy. Immature HBV in the cell collects nucleotides in the capsid and uses them to perform reverse transcription to produce DNA. During this process, the nucleotides in the capsid do not use external sources of energy, such as ATP, and instead spontaneously follow a free energy gradient to enter the capsid. The capsid shell and pgRNA are ionized and covered with negative charges. Negatively charged nucleotides enter the capsid easily despite the negatively charged capsid shell and pgRNA inside it.

Some of the most commonly used antiviral reagents are nucleotide analogs. They enter the capsid, connect with a polymerase inside the capsid, and inhibit reverse transcription. These reagents include entecavir, lamivudine, and adefovir. They must permeate the capsid shell to efficiently reach the polymerase. The permeation mechanism should be similar between the nucleotides and reagents. Recently, viruses resistant to these drugs have been identified, and a need to develop new antiviral reagents has emerged. Thus, understanding the drug delivery mechanism inside the HBV capsid has become very important, namely the physico-chemical mechanism that generates the free energy gradient that makes it possible for the HBV capsid to efficiently collect nucleotides and reagents.

Physico-chemical studies of HBV have focused on the capsid structure based on electron microscope measurements. ${ }^{2-7} \mathrm{~A}$ detailed three-dimensional crystal structure was obtained by Zlotonic et al., ${ }^{7}$ who reported that the pores along the 5-fold rotational symmetry axis are small, while the pores along the 2-fold and 3-fold rotational symmetry axes are large enough for nucleotides to pass through. Thus, from a geometrical viewpoint, the nucleotides are believed to enter the capsid through the pores along the 2-fold or 3-fold rotational symmetry axes, and the surfaces of these pores are hydrophilic and hydrophobic, respectively. Because the chemical environment differs in these pores, we cannot discuss the capture of nucleotides solely in terms 
of pore size. Wynne et al. investigated the mechanical properties of the HBV capsid based on atomic force microscopy (AFM) measurements and reported that the HBV capsid is not as hard as that of other viruses, but it is robust and flexible enough to allow for reverse transcription to occur inside it. ${ }^{8}$ Thus, although the HBV capsid has been investigated, the field in which HBV exerts its surroundings has yet to be determined.

All-atom molecular dynamics (AA-MD) simulations are a powerful tool for studying viruses in solution. Many AA-MD simulations of viruses have been conducted. For example, small viruses such as small satellite tobacco mosaic viruses, ${ }^{10}$ satellite tobacco necrosis virus, ${ }^{11}$ and porcine circoviruses ${ }^{12}$, but also larger viruses ${ }^{13}$ such as polioviruses, hepatitis B viruses (HBV), ${ }^{14,15}$ and immunodeficiency virus ${ }^{16,17}$, have been investigated. Hadden et al. ${ }^{14}$ investigated the physical properties of an HBV without RNA, that is, with an empty capsid, in an aqueous electrolyte solution. They observed asymmetric structural fluctuations in the HBV capsid, and reported that the permeation of sodium ions was faster than that of chloride ions. The authors noted that this difference may be related to the permeation of nucleic acids across the HBV capsid shell. However, the microscopic origin of the difference in the permeation rates remains unclear.

The purpose of the present study was to examine the microscopic physicochemical environment of negatively charged substance, such as nucleotides and antiviral reagents, that approach the surface of negatively charged HBV capsids and penetrate the interior, where negatively charged pgRNA is present. We performed a long AA-MD calculation for an entire HBV capsid containing pgRNA; the capsid was in solution. Electric field analysis of the resultant trajectory showed how negatively charged particles approach the surface of the HBV capsid. The free energy profiles of water molecules and ions were calculated as functions of the distance from the center of mass of the HBV. Pore selectivity by the water molecules and ions was also explored.

The MD calculations are presented in the Methods section, followed by the Results and Discussion section, before a final overview of the physical properties of HBV with pgRNA in the Conclusion section.

\section{METHODS}

AA-MD simulations were performed for an immature $\mathrm{HBV}$ with pgRNA to investigate the physical properties of the virus in the electrolyte solution at the molecular level. The system was modeled as discussed in the following sections. 


\section{A. Capsid}

The HBV capsid consists of $240 \mathrm{HBc}$ proteins. The HBc protein consists of 183 residues and has the following three regions: ${ }^{18}$ (1) residues 1-140, which comprise the N-terminal domain (NTD) that forms the capsid structure; (2) residues 150-183, which comprise the arginine-rich Cterminal domain (CTD) that forms the inner surface of the capsid that attaches to pgRNA and polymerase; (3) the remaining residues 141-149 are known as linkers, which attach to the NTD and CTD. Although the amino acid sequence was the same among the three domains, they were classified into four conformationally different types: chains A, B, C, and D. The capsid is an icosahedron fabricated by 60 subunits, each composed of a set of A, B, C, and D chains. We produced the HBV capsid assuming icosahedral symmetry, as determined by Hong Zhou (PDB ID: $3 \mathrm{~J} 2 \mathrm{~V}) .{ }^{5}$ Then, we modified its structure since several unstable geometries were found in the original PDB data. Furthermore, SER157, SER164, and SER172 were initially assumed to be phosphorylated. However, a recent study ${ }^{19}$ found that several phosphorylated amino acids are dephosphorylated when pgRNA is included in the HBV capsid, although the number of dephosphorylated amino acids has not yet been determined quantitatively. It has also been suggested that a phosphorylated CTD stabilizes the capsid. ${ }^{20}$ Despite several observations, the phosphorylation state of the amino acids has yet to be fully elucidated. In our MD system, all SER157, SER164, and SER172 in the CTD were phosphorylated, since the phosphorylated structure was considered to be the most stable. The protonation of each amino acid was determined randomly according to the probability of protonation. The probabilities were calculated according to the $\mathrm{pKa}$ values in the solvent at $\mathrm{pH}$ 7.2. For more details, see the Supporting Information.

In general, pgRNA of immature HBV does not form a specific 3D structure, ${ }^{3}$ but it partially forms secondary structures. ${ }^{21,22}$ Thus, we prepared pgRNA with partial secondary structures and inserted it into the HBV capsid. Details are presented in the Supporting Information section.

\section{B. Capsid in electrolyte solution}

TABLE I. System in a MD unit cell.

\begin{tabular}{cc}
\hline Components & $\begin{array}{c}\text { Number of } \\
\text { molecules }\end{array}$ \\
\hline $\begin{array}{c}\text { Capsid } \\
\text { protein }\end{array}$ & 240 \\
\hline
\end{tabular}




\begin{tabular}{cc}
\hline pgRNA & 1 \\
Water & $2,382,166$ \\
$\mathrm{Mg}^{2+}$ & 138 \\
$\mathrm{Na}^{+}$ & 613 \\
$\mathrm{~K}^{+}$ & 8,586 \\
$\mathrm{Cl}^{-}$ & 6,465 \\
\hline Total atoms & $7,995,566$ \\
\hline
\end{tabular}

The degree of hydration of the protein was determined using a dowser. ${ }^{23}$ The water molecules were inserted into the MD cell using the solvate plugin of Visual Molecular Dynamics (VMD). ${ }^{24} \mathrm{Na}^{+}, \mathrm{K}^{+}$, and $\mathrm{Cl}^{-}$ions were added by replacing water molecules. To avoid ion concentration gradients in the system, the MD cell was divided into three regions according to their distance $(R<8 \mathrm{~nm}, 8 \mathrm{~nm}<R<16 \mathrm{~nm}$, and $16 \mathrm{~nm}<R)$ from the center of mass of the capsid, and the above ion insertion was performed in each region. Ion insertion was performed using the Autoionize plugin of VMD. Since $\mathrm{Mg}^{2+}$ ions are believed to stabilize RNA significantly, ${ }^{25,26}$ the ions were placed around the pgRNA. Our ion concentrations were in good agreement with the experimental intracellular ion concentrations. ${ }^{27}$ The number of molecules in the MD unit cell is summarized in Table I. The total number of atoms was approximately eight million.

\section{Equilibration of the system}

Three-step MD calculations were initially carried out to equilibrate the system. First, MD calculations were performed for $3 \mathrm{~ns}$ in the NVT ensemble while constraining the positions of the $\mathrm{C}_{\alpha}$ atoms of the capsid and the radial distribution of the pgRNA around the capsid, with its partial secondary structures preserved to avoid any structural fracture in the capsid or pgRNA. Second, the constraints were removed step-by-step. This MD calculation was performed in the NVT ensemble at $T=310.15 \mathrm{~K}$. The temperature was maintained using a velocity-scaling thermostat. The MD system was spatially divided into 64 regions $(4 \times 4 \times 4)$ and an independent thermostat was adapted for each region. Finally, a 6-ns MD calculation was performed in the NVT ensemble at $T=310.15 \mathrm{~K}$ using a five-chain Nosé-Hoover thermostat. ${ }^{28}$

Then, a $100 \mathrm{~ns} \mathrm{MD}$ calculation was performed in the $N P T$ ensemble at $P=0.1 \mathrm{MPa}$ and $T=$ $310.15 \mathrm{~K}$ to reach thermal equilibration. This was followed by a further 100 -ns production calculation for the analysis. The pressure was controlled using an Andersen barostat, ${ }^{29}$ and the temperature was controlled using a five-chain Nosé-Hoover thermostat. 


\section{MD calculation}

The CHARMM36 potential set was used for the proteins, ${ }^{30}$ pgRNA, ${ }^{31}$ and ions ${ }^{32}$, while CHARMM-modified TIP3 $\mathrm{P}^{33}$ was used for the water molecules. The cutoff distance was set at $1.2 \mathrm{~nm}$ for the short-range Lennard-Jones interaction, with a switching function from 1.0 to 1.2 $\mathrm{nm}$. The long-range Coulombic interaction was calculated using the fast multipole method $(\mathrm{FMM})^{34,35}$ with a spherical harmonic expansion up to the fourth order, and the interaction with the multipole moments of the entire MD cell in the periodic boundary condition was calculated using the Ewald method. The barostat and thermostat were coupled every $0.5 \mathrm{ps}$. The equation of motion was integrated using the RESPA ${ }^{36}$ multi-time-step algorithm with $\Delta t=0.5 \mathrm{fs}$ for the intramolecular interactions, $\Delta t=2 \mathrm{fs}$ for the pair interactions, and $\Delta t=4 \mathrm{fs}$ for the FMM. With respect to the chemical bonds that involved hydrogen atoms, the bond length was constrained using SHAKE/ROLL and RATTLE/ROLL. ${ }^{37}$ The calculations were carried out on the Kcomputer $^{38}$ using MODYLAS ${ }^{34}$ developed by our group.

\section{E. Pore size}

The capsid has four kinds of pores around the 2-fold, 3-fold, pseudo-3-fold, and 5-fold rotational symmetry axes. However, the shape of the pores is not a simple straight cylinder. To characterize the shape of the pores, the pore size $r_{\text {pore }}(R)$ was defined as the distance between the rotational symmetry axis and the closest atom of the NTD. The size was calculated as a function of the distance $R$ along the rotational symmetry axis from the center of mass of the HBV capsid.

\section{Rotational Symmetry Axis}

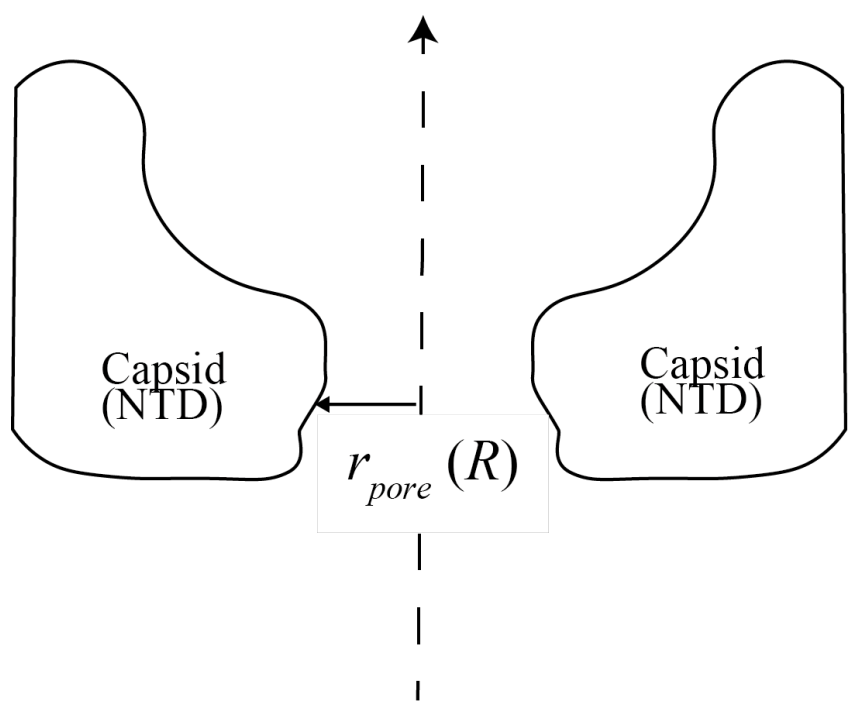


FIG. 1. Schematic diagram of the cross section of the rotational symmetry axis of the capsid.

The pore size $r_{\text {pore }}$ is a function of the distance $R$ from the center of mass of the HBV capsid.

\section{F. Free energy profile of the water molecules and ions}

The free energy profiles of the water molecules and various ions were calculated along the trajectory when they passed through the pores and entered the HBV capsid. A free energy profile can be calculated as a function of the distance $R$ from the center of mass of the capsid, as follows:

$\Delta G(R)=k_{b} T \ln \frac{\rho(R)}{\rho_{0}}$

where $k_{b}$ and $T$ are the Boltzmann constant and system temperature, respectively. $\rho(R)$ is the number density of the water molecules or ions. The reference density, $\rho_{0}$, was taken to be $\rho(R)$ at $R=20 \mathrm{~nm}$, where is the bulk aqueous solution region out of the capsid. To calculate $\rho(R)=$ $N(R) / V(R)$, we evaluated the number of water molecules or ions, $N(R)$, and the volume $V(R)$ of the space where water molecules and ions are accommodated. We measured a cylinder of radius of $2 \mathrm{~nm}$ centered on the rotational symmetry axis and sliced this cylinder into sections with a width of $0.15 \mathrm{~nm}$ in the direction of the rotational symmetry axis (side view in Fig. 2). The $N(R)$ in each section is calculated. Next, we divided this section into meshes of $0.05 \times 0.05 \mathrm{~nm}$ (top view in Fig. 2). $V(R)$ was calculated by summing the volumes of the meshes, excluding the meshes occupied by the capsid or RNA atoms. Here, the atoms were considered to be spheres with a radius of $0.15 \mathrm{~nm} . V(R)$ is the volume that follows the shape of the pores.

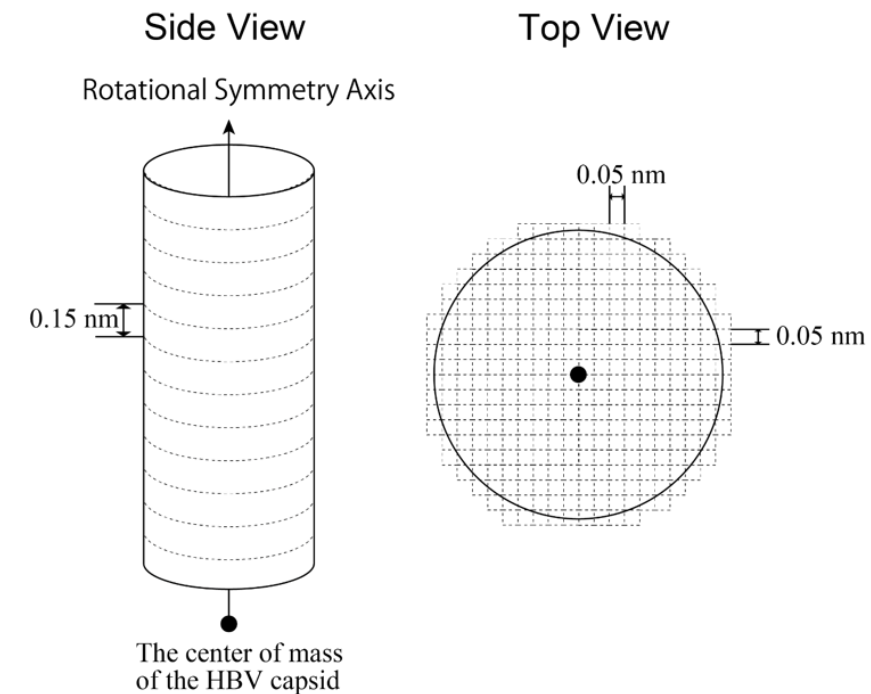

FIG. 2. Illustration of the volume profile calculation along the rotational symmetry axis as a function of distance $R$ from the center of mass of the HBV capsid. 
The free-energy profile, described by Eq. (1), is a simple function of the radial direction, $R$, from the center of mass of the capsid. In other words, it is the free energy averaged in the latitude and longitude directions within the pore. Therefore, although heterogeneity in the lateral direction within the pore is ignored in the analysis, the free energy profile of small molecules along the HBV capsid pore can be effectively discussed.

\section{RESULTS AND DISCUSSION}

\section{A. Equilibration calculation}

The root mean square deviation (RMSD) was calculated for the backbone atoms of the amino acid residues of the capsid, except for the CTD and the loop, which are known to have disordered structures. The RMSD was also calculated for the pgRNA nucleic acids. In addition to these RMSD values, the number of hydrogen bonds and the distance from the center of mass of the capsid to the top of the spike were also calculated to examine equilibration. The RMSDs are shown in Fig. 3. The RMSD for the capsid converged to an equilibrium value of $0.455 \mathrm{~nm}$ in 100 ns. It was on the same order of magnitude as that of ordinary proteins. This implies that the capsid structure reached a stable structure. In contrast, the RMSD of the pgRNA continued to increase during the entire calculation period of $200 \mathrm{~ns}$. This result suggests that the pgRNA is weakly bonded to the C-terminus of the capsid and that the pgRNA structure may not be strongly constrained. As a result, pgRNA can move freely. The cryo-EM experiment showed that the pgRNA in the HBV capsid does not have a specific structure. The present calculation is in good agreement with the experimental results.

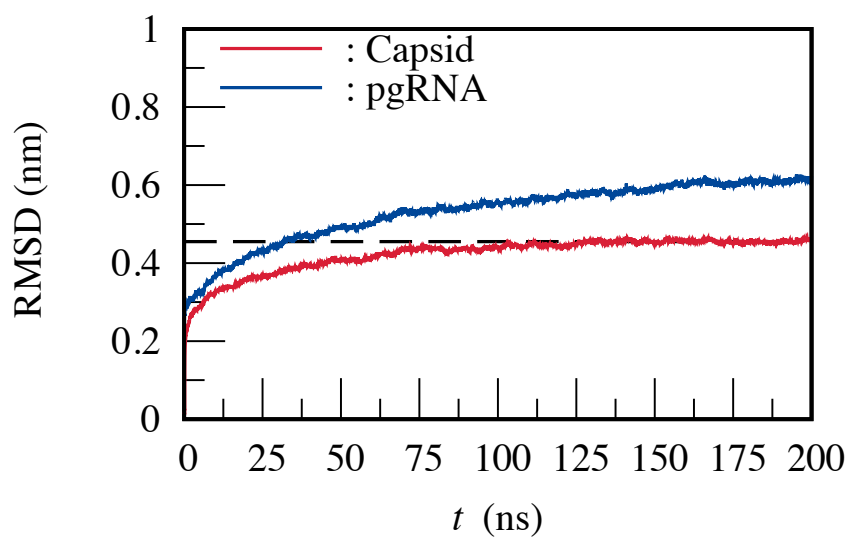

FIG. 3. RMSDs of the HBV capsid (red) and pgRNA (blue) as functions of time. 


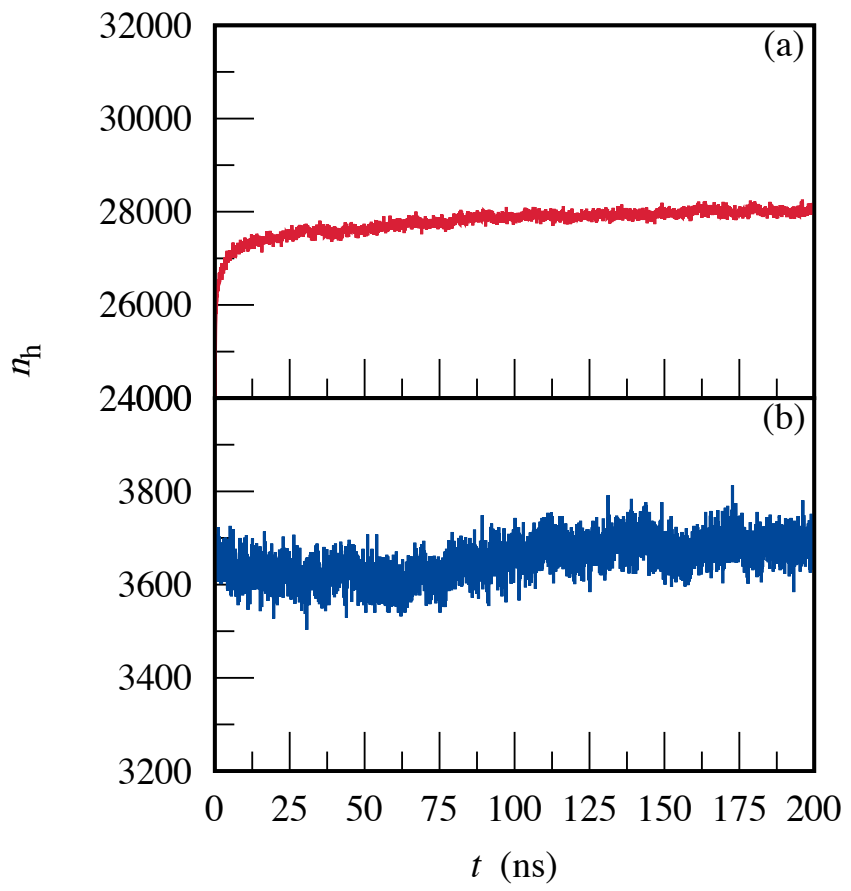

FIG. 4. The number of hydrogen bonds of (a) the HBV capsid and (b) the pgRNA as functions of time.

An equilibration run of $100 \mathrm{~ns}$ was required until the number of hydrogen bonds in the HBV capsid converged to an equilibrium value. The structural stability of the HBV capsid increased as the number of hydrogen bonds increased. The calculated number of hydrogen bonds in the pgRNA was almost constant during the MD calculation; that is, the pgRNA structure remained stable.

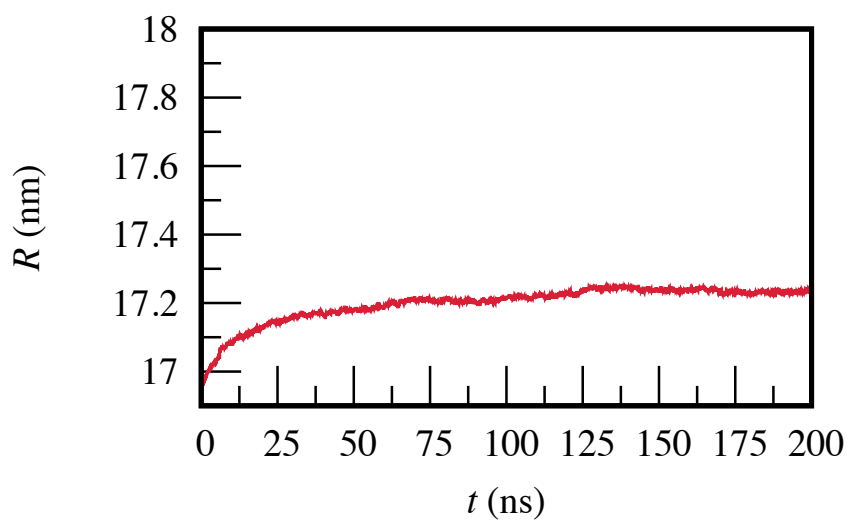

FIG. 5. Distance from the center of mass to the top of the HBV spike.

The distance from the center of mass of the HBV capsid to the top of the spike (RESID: 7680) converged to $17.2 \mathrm{~nm}$ after $100 \mathrm{~ns}$. Thus, our calculated capsid radius was $17.2 \mathrm{~nm}$, which is 
in agreement with the experimental value of $17.4-18.0 \mathrm{~nm} .^{2,7}$ The function shows good convergence to a stable structure of the capsid during our equilibration run.
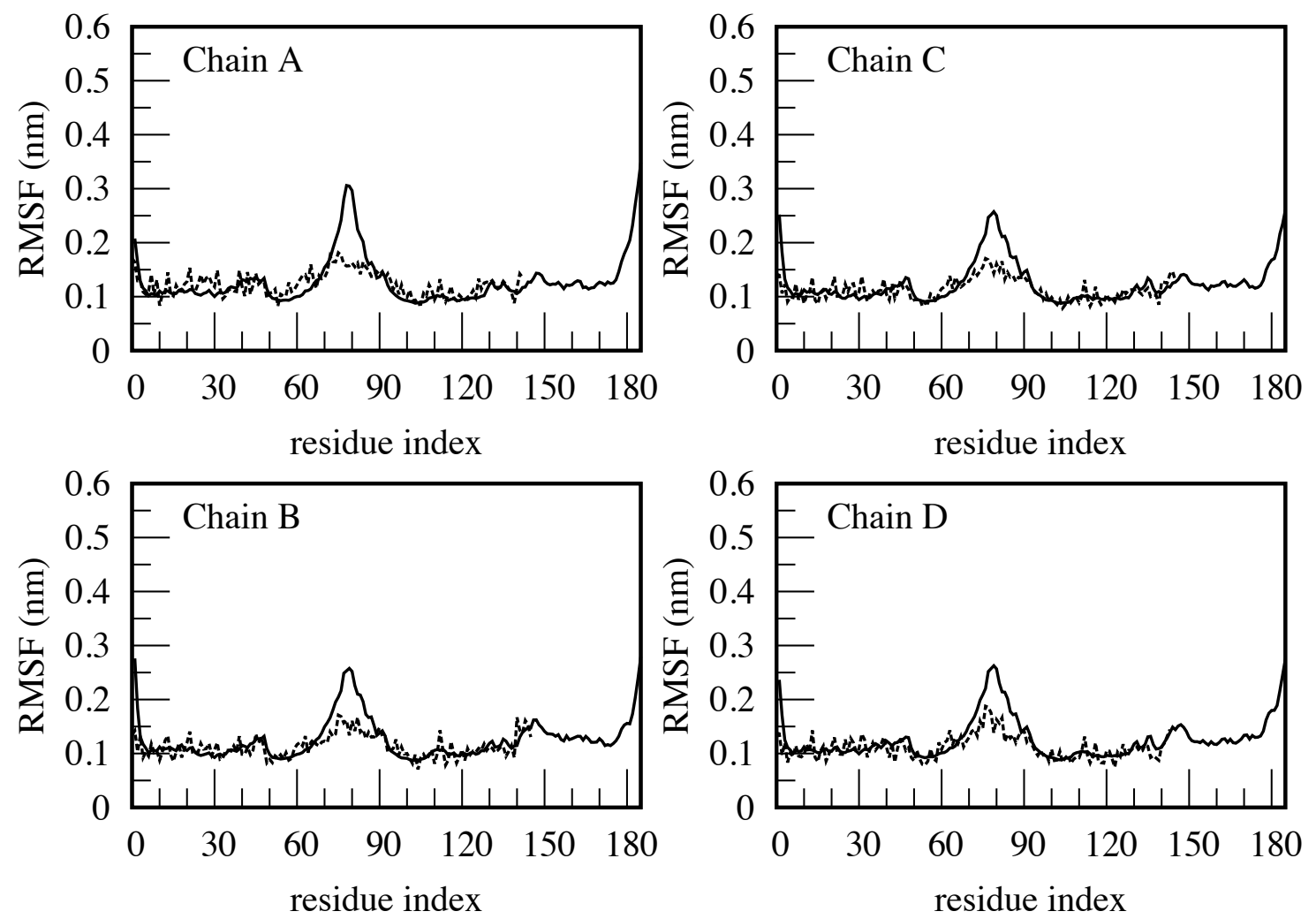

FIG. 6. The calculated (solid line) and experimental (dotted line) RMSFs of the $\alpha$-carbon of each chain.

The root mean square fluctuation (RMSF) of the $\alpha$-carbons of each chain is shown in Fig. 6. The fluctuation was averaged over the last $100 \mathrm{~ns}$ of the trajectories. The experimental RMSF shown in Fig. 6 was calculated using the equation, ${ }^{39} \operatorname{RMSF}\left(B_{i}\right)=\frac{\sqrt{B_{i}}}{2 \sqrt{2} \pi}$, where $\operatorname{RMSF}\left(B_{i}\right)$ and $B_{i}$ are the experimental RMSF and Cryo- $\mathrm{EM}^{5}$ temperature factors of the $i$-th $\alpha$-carbon, respectively. The value of the experimental RMSF was smaller than the calculated value because the temperature factor was measured at extremely low temperatures $(77 \mathrm{~K})$. Despite this difference, the peak positions of our RMSF were consistent with the experimental positions. Thus, we succeeded in reproducing the capsid structure, including the fluctuating motion of the capsid atoms. The RMSFs of the N-terminals (RESID: 1-4), spikes (RESID 65-95), and C-terminals (RESID: 150-183) were larger than those in other regions. While the HBV capsid was relatively hard with smaller fluctuations in solution, the terminals and spikes exhibit larger fluctuations. Hadden et al. ${ }^{14}$ reported similar results. 


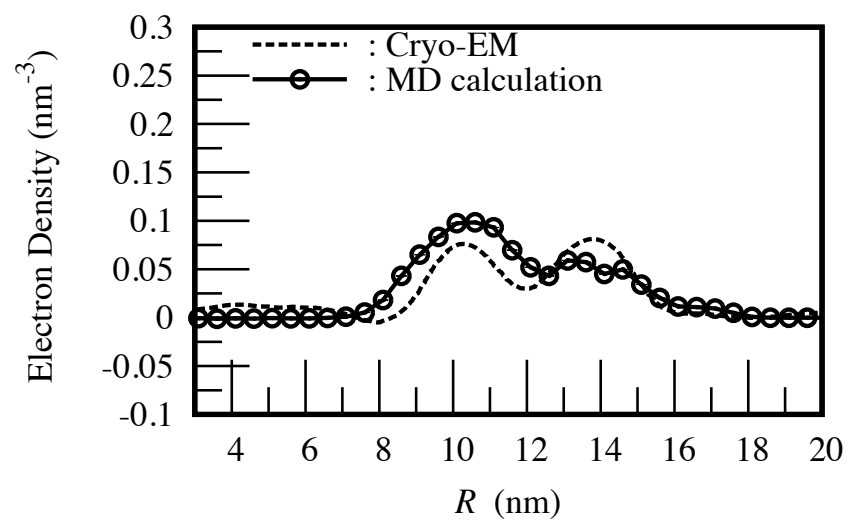

FIG. 7. The radial electronic density profile as a function of the distance from the center of mass of the HBV capsid. The solid and dotted lines denote the calculated and experimental results, ${ }^{2}$ respectively.

Fig. 7 shows the calculated and experimental radial electronic density profiles as functions of the distance from the center of mass of the HBV capsid. Both the experimental and calculated profiles exhibited two peaks at the same position. However, the height of the distribution differed slightly between the two. The experimental values had an error of approximately $1.4-2.0 \mathrm{~nm}$ on the horizontal axis. ${ }^{2}$ Furthermore, the calculation was the average of $100 \mathrm{~ns}$ for a single HBV. Considering these factors, we can conclude that the experimental and calculated electron densities do not contradict each other within the error. The peak at $8<R<12 \mathrm{~nm}$ had a large contribution from pgRNA and CTD, and the peak at $12<R<16 \mathrm{~nm}$ was also attributable to the NTD.

In any case, as discussed above, the system reached thermal equilibrium after a $100 \mathrm{~ns}$ MD run, and the RMSF and radial electronic density profile were in qualitative agreement with the experimental results. In the following analysis, the trajectories observed after 100 ns were examined. An image of the equilibrated system is shown in Fig. 8. 


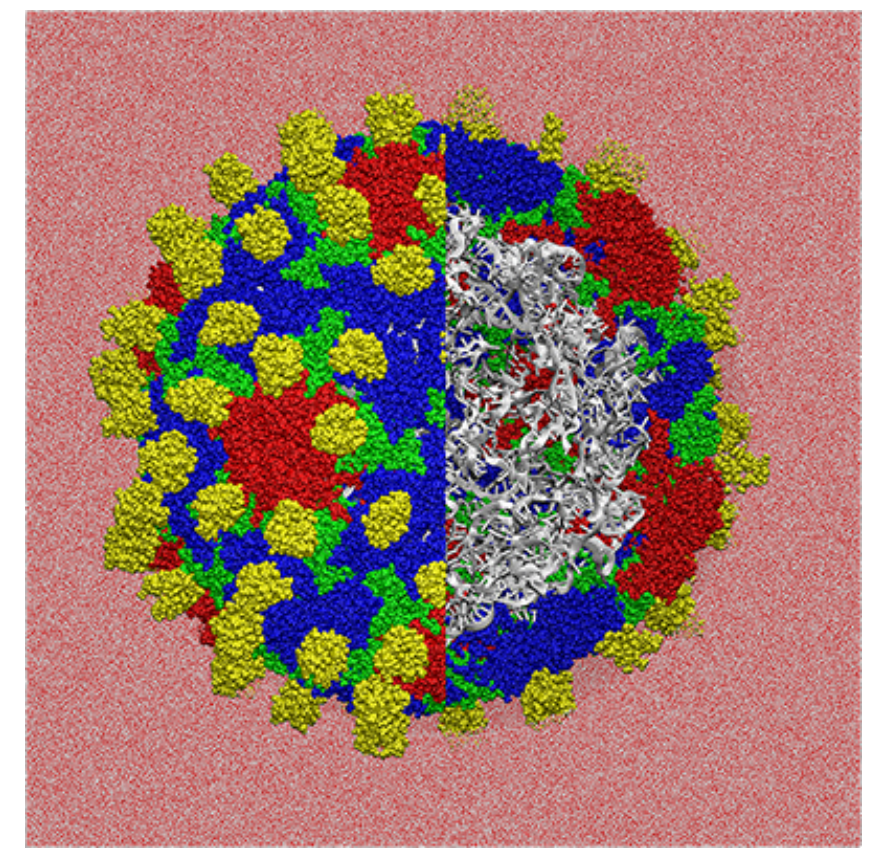

FIG. 8. The entire HBV after a 100-ns equilibration calculation. The capsid is shown in red, green, blue, and yellow. The white lines in the capsid represent pgRNA. The small red and white spheres in the background indicate water molecules. The right half front of the capsid proteins were omitted to show the inner structure of the capsid.

\section{B. Electric field}

The HBV capsid without the CTDs were charged by negative electricity of $-1535 \mathrm{e}$. The pgRNA in the HBV capsid were also charged negatively by -3396e. Despite the negative charges in the capsid and pgRNA, nucleotides, which are also negatively charged, were found to approach and enter the capsid and bond with the polymerase near the pgRNA. Therefore, we analyzed the electric field produced by the capsid and pgRNA to determine the mechanism by which the nucleotides were able to approach and enter the HBV capsid.

The electric field was calculated in three dimensions. The field was approximately obtained from the coulombic force acting on each atom of the water molecule divided by its electric charge. This corresponds to the measurement of the electric field using the atomic charge of the water molecule as a probe. Furthermore, the calculated electric field was further averaged by utilizing the icosahedral symmetry of the capsid to obtain a high accuracy. Fig. 9 shows a diagram of the geometrical relationship between the capsid structure and rotational symmetry axis. The electric field was measured by the probe located at a distance $R$ from the center of mass of the capsid, and Fig. 10 shows the measured electric field. This value is directly related to the force acting on negatively charged substances, such as nucleotides and drugs above the capsid. The red and blue 
colors represent the outward (repulsive) and inward (attractive) forces, respectively. The white region is the region where negligibly weak or zero electrostatic forces act between the capsid and negatively charged particles.

(a)

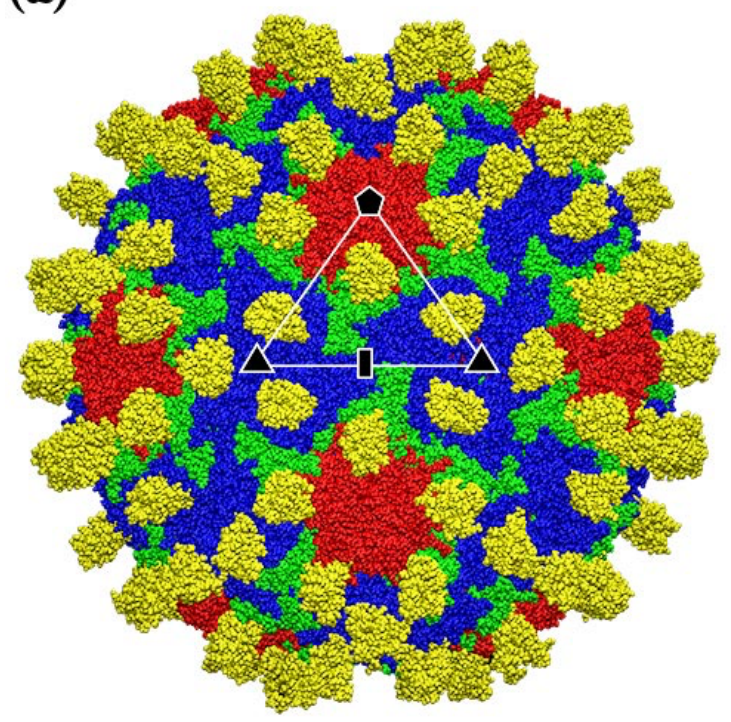

(b)

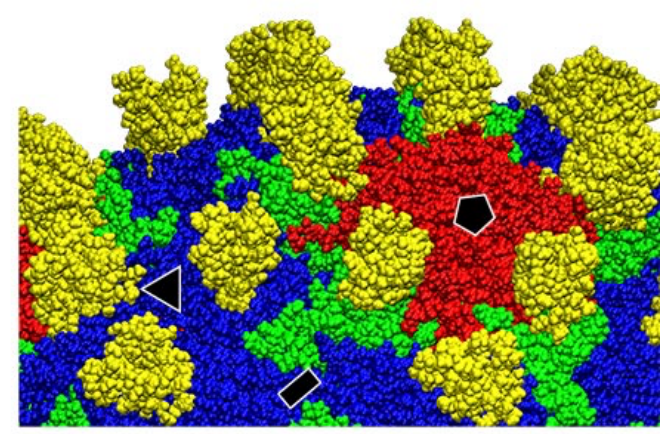

(c)

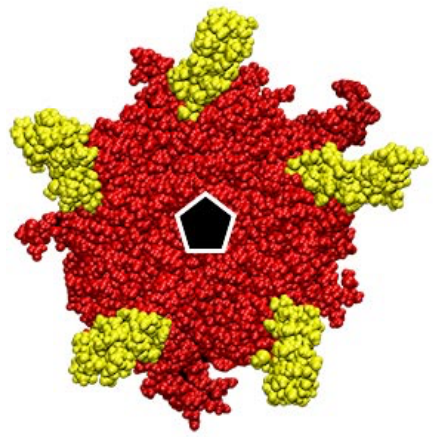

5-fold axis

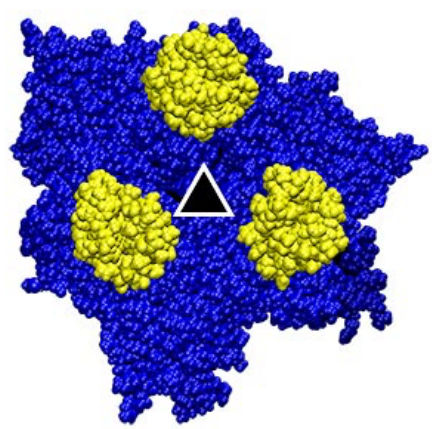

3-fold axis

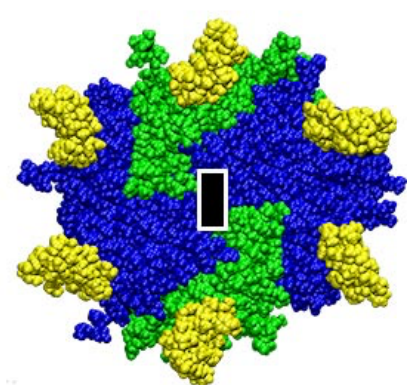

2-fold axis

FIG. 9. (a) The entire capsid and symmetry axis. Yellow indicates the spikes of the capsid (ResID: 63-94). The pentagons, triangles, and rectangles shown in black represent the positions of the 5-, 3-, and 2-fold rotational symmetry axes, respectively. (b) Enlarged view around the 5-, 3-, and 2fold rotational symmetry axes. The red, green, and blue areas indicate the floor region. (c) The 5-, 3-, and 2-fold rotational symmetry axes. 

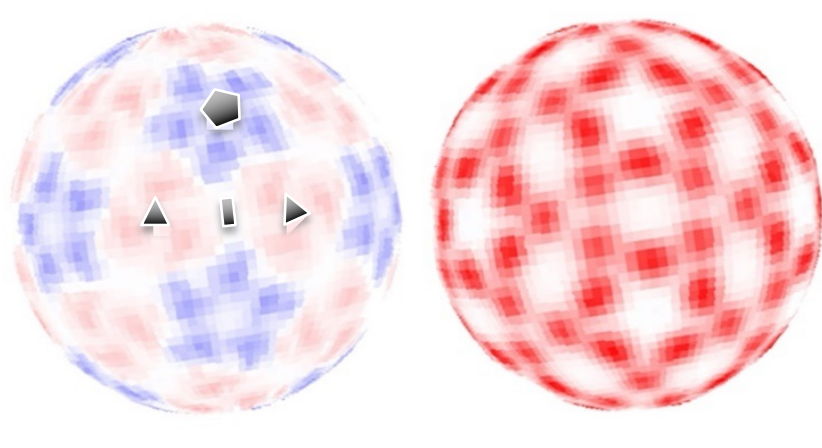

$R=17 \mathrm{~nm}$

$$
R=18 \mathrm{~nm}
$$

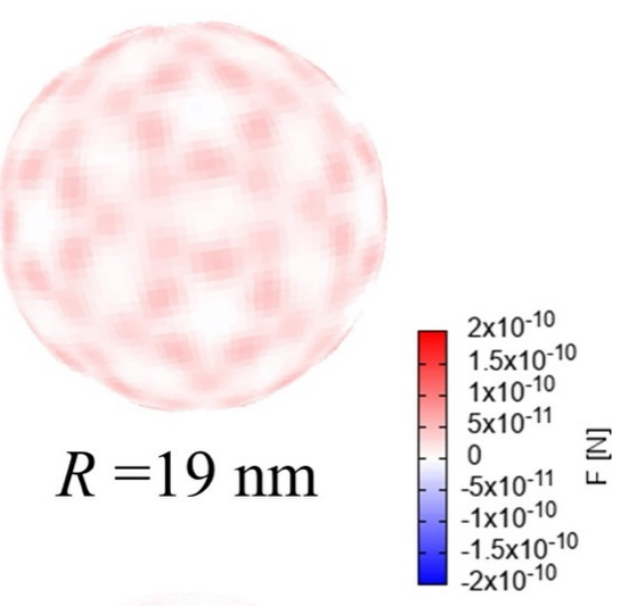

$R=20 \mathrm{~nm}$

$R=21 \mathrm{~nm}$

$R=21.5 \mathrm{~nm}$

FIG. 10. The averaged electrostatic field produced by the HBV capsid in solvent expressed in units of force acting on a negatively charged particle with -e measured over the spheres with several different radii $R$ around the capsid. Red and blue indicate the outward (repulsive) and inward (attractive) forces, respectively. White indicates a negligibly weak or zero force.

Fig. 10 shows that at $R=21.5 \mathrm{~nm}$ from the capsid, an almost zero mean electric force acts on a negatively charged particle in solution. At $R=20 \mathrm{~nm}$, the force certainly acts, although it is negligibly weak. These zero or very weak forces may be understood when we consider the Debye length of the present electrolyte solution $(150 \mathrm{mM})$, which is $0.77 \mathrm{~nm}$. At $R=20 \mathrm{~nm}$, the distance measured from the tip of the capsid spike was $2.8 \mathrm{~nm}$. This is 3.6-times the Debye length. The shielded force has little effect on the motion of negatively charged substances, such as nucleotides and antiviral reagents. In other words, the negatively charged particles cannot recognize the HBV capsid at a distance further than $20 \mathrm{~nm}$, and the particles can approach the capsid by a random walk. 
Next, at $18<R<20 \mathrm{~nm}$ in the region above the spike tip, repulsive forces were found to act on the negatively charged particles in solution. This is because negatively charged residues are present on the top of the spike (D77, D78, E83). In contrast, in the regions between the spike tips, the forces acting on the negatively charged particles, were still negligibly weak, and negative ions were able to approach the capsid by random walks, avoiding the spikes.

At $R=17 \mathrm{~nm}$, above the floor, attractive forces act on the negative charges. The attractive force areas exist around a pore along the 5-fold rotational symmetry axis located on the floor. Ten positively charged residues, ARGs (RESID: R127, R133), exist around the axis of symmetry. Negative ions thus tend to come around the 5-fold rotational symmetry axis. The attractive force was weak, ca. $-0.5 \times 10^{-10} \mathrm{~N}$. However, if this small force acts over a range of $1 \mathrm{~nm}$, from $R=$ 18 to $17 \mathrm{~nm}$, then the free energy of transfer of the negative charge to the capsid surface is approximately $-30 \mathrm{~kJ} / \mathrm{mol}$, which is very large. Thus, negatively charged substances tend to gather around the 5-fold rotational symmetry axes of HBV. Furthermore, in Fig. 10 for $R=17$ $\mathrm{nm}$, the white area spreads over the entire surface of the capsid, except for the areas surrounding the 3-fold symmetry axis, where a small electrostatic force acts on the negatively charged particles. This implies that negatively charged substances can migrate freely over the floor of the capsid. Migrating substances can find the pores of HBV.

In summary, negatively charged substances, such as nucleotides and antiviral reagents, can approach the HBV capsid by thermal motion, avoiding the spikes. The substances then migrate all over the floor of the HBV capsid. Finally, they find pores through which they can pass through the HBV capsid shell. In the next section, we investigate the properties of the pores related to the permeability of the substances to the free energy profile of water molecules and ions.

\section{Exchange of water molecules and ions across the capsid shell}

Water molecules and ions can enter and exit the HBV capsid through the capsid pores. The number of water molecules and ions moving from the inside to the outside or from the outside to the inside of the capsid per $1 \mathrm{~ns}$ was calculated, and the data are shown in Table II, together with results reported previously for other viruses. In our calculation, specifically, water molecules or ions that existed within $R=13.6 \mathrm{~nm}$ but were found outside of the capsid $(R>14.0 \mathrm{~nm})$ after 1 ns were considered to have permeated from the inside to the outside. Likewise, the water molecules or ions that existed outside of the capsid with $R>14.0 \mathrm{~nm}$ but were found inside of the capsid with $R<13.6 \mathrm{~nm}$ after $1 \mathrm{~ns}$ were counted as molecules that permeated from the outside to the inside. 
TABLE II. The calculated permeation rates of water molecules and ions between the inside and outside of viral capsids.

\begin{tabular}{lccccc}
\hline Virus & $\begin{array}{c}\text { Components } \\
\text { studied }\end{array}$ & $\begin{array}{c}\text { Water } \\
\left(\mathrm{ns}^{-1}\right)\end{array}$ & $\begin{array}{c}\mathrm{K}^{+} \text {ion } \\
\left(\mathrm{ns}^{-1}\right)\end{array}$ & $\begin{array}{c}\mathrm{Cl}^{-} \text {ion } \\
\left(\mathrm{ns}^{-1}\right)\end{array}$ & $\begin{array}{c}\mathrm{Na}^{+} \text {ion } \\
\left(\mathrm{ns}^{-1}\right)\end{array}$ \\
\hline HBV (inward) & Capsid/pgRNA & $3200 \pm 250$ & $11 \pm 1$ & $1.4 \pm 0.2$ & $0.6 \pm 0.1$ \\
HBV (outward) & Capsid/pgRNA & $3200 \pm 250$ & $10 \pm 1$ & $1.1 \pm 0.3$ & $0.7 \pm 0.3$ \\
\hline \multicolumn{7}{c}{ Reported studies } \\
\hline Polio virus $^{13}$ & Empty capsid & $8 \pm 2$ & 0 & 0 & 0 \\
PCV2 $^{12}$ & Empty capsid & $73 \pm 3$ & - & 0 & 0 \\
STNV $^{11}$ & Empty capsid & 10 & - & - & - \\
HIV-1 $^{17}$ & Empty capsid & $20100 \pm 1600$ & - & $9.4 \pm 2.4$ & $22.2 \pm 2.9$ \\
Empty HBV $^{14}$ & Empty capsid & $4700 \pm 0.04$ & - & $1.8 \pm 0.4$ & $8.4 \pm 0.7$ \\
\hline
\end{tabular}

HBV: hepatitis B virus

PCV2: porcine circovirus type 2

STNV: satellite tobacco necrosis virus

HIV: human immunodeficiency virus

The calculated permeation rates of water molecules from the outside to the inside and from the inside to the outside were $3200 \mathrm{~ns}^{-1}$. The permeation rates of $\mathrm{Na}^{+}, \mathrm{K}^{+}$, and $\mathrm{Cl}^{-}$ions from the outside to the inside of the capsid were $11 \pm 1 \mathrm{~ns}^{-1}, 1.4 \pm 0.2 \mathrm{~ns}^{-1}$, and $0.6 \pm 0.1 \mathrm{~ns}^{-1}$, respectively. Similarly, those from the inside of the capsid to the outside were $10 \pm 1 \mathrm{~ns}^{-1}, 1.1 \pm 0.3 \mathrm{~ns}^{-1}$, and $0.7 \pm 0.3 \mathrm{~ns}^{-1}$, respectively. The permeation rates were similar in both directions. These results clearly show that the exchange of water and ions between the inside and outside of the capsid occurred in thermal equilibrium. The permeation rate of potassium ions was 16 times higher than that of sodium ions. This was caused by the difference in the concentration. The concentration of potassium ions was 14 times higher than that of the sodium ions. Therefore, to compare the permeation rates of these two types of ions, we normalized the rates by the ionic concentrations. Thus, the quantity in the table should be normalized to the permeation rate of ions per $1 \mathrm{~mol} / \mathrm{dm}^{3}$. The concentrations of potassium ions, chloride ions, and sodium ions in the present system were $140 \mathrm{mM}, 150 \mathrm{mM}$, and $10 \mathrm{mM}$, respectively. The normalized rates were $71 \mathrm{~ns}^{-1} \cdot\left(\mathrm{mol} / \mathrm{dm}^{3}\right)^{-1}, 8.7$ $\mathrm{ns}^{-1} \cdot\left(\mathrm{mol} / \mathrm{dm}^{3}\right)^{-1}$, and $60 \mathrm{~ns}^{-1} \cdot\left(\mathrm{mol} / \mathrm{dm}^{3}\right)^{-1}$ for potassium ions, chloride ions, and sodium ions, respectively. The values for positively charged potassium and sodium ions were almost the same. In contrast, the value for the negatively charged chloride ions was approximately eight times smaller than that of the positively charged ions. Therefore, the HBV capsid exhibits ion selectivity 
when it captures the ions inside it. Hadden et al ${ }^{14}$ also reported that the permeation rate of chloride ions in an empty HBV was five times slower than that for sodium ions. Whether the pgRNA is contained in the capsid, the positively charged substances can move in and out of the HBV capsid faster than negatively charged substances. This is because several residues prevent negatively charged particles from passing through the pores of the capsid. This will be discussed in detail in the following sections.

Although it is difficult to compare these values for different viruses due to size differences, we can qualitatively discuss the exchange of water and ions between the interior and exterior of the viruses. The permeation rates of water and ions for the viruses reported thus far are summarized in Table II. Three viruses, poliovirus, porcine circovirus type 2 (PCV2), and satellite tobacco necrosis virus (STNV), do not have envelopes, while human immunodeficiency virus (HIV-1) and HBV have envelopes. The permeation rates of water for empty poliovirus, empty PCV2, and empty STNV were 8, 73, and $10 \mathrm{~ns}^{-1}$, respectively. The ions did not enter or exit these three virus capsids. In contrast, the exchange rates of water for HIV-1 and empty HBV were 20,100 and $4,700 \mathrm{~ns}^{-1}$, the permeation rates of sodium ions were 22.2 and $8.4 \mathrm{~ns}^{-1}$, and the rates of chloride ions were 9.4 and $1.8 \mathrm{~ns}^{-1}$, respectively. Our calculations also show that the permeation rates of water molecules and ions through the present HBV with pgRNA are of the same order of magnitude as those reported previously for empty HIV-1 and HBV and greater than those of poliovirus, PCV2, and STNV. Fewer water molecules or ions can pass through poliovirus, PCV2, and STNV because they do not have large pores in their capsids. Since these viruses do not have an envelope, they most likely have small pores that limit the permeation of water and ions to maintain a constant condition inside the viral capsid. In contrast, the large pores in the capsids of HBV and HIV-1 allow many water molecules and ions to pass through their capsids. We can consider that the latter viruses may have large pores because they have an envelope that protects the capsid, keeping its environment separate from the external solution.

\section{Pore sizes and free energy profiles of water molecules and ions}

Pores play a key role in the penetration of water and ions across the capsid shell. Here, we analyzed the size distribution of the pores and the free energy profiles of water molecules and ions permeating through the pores. 


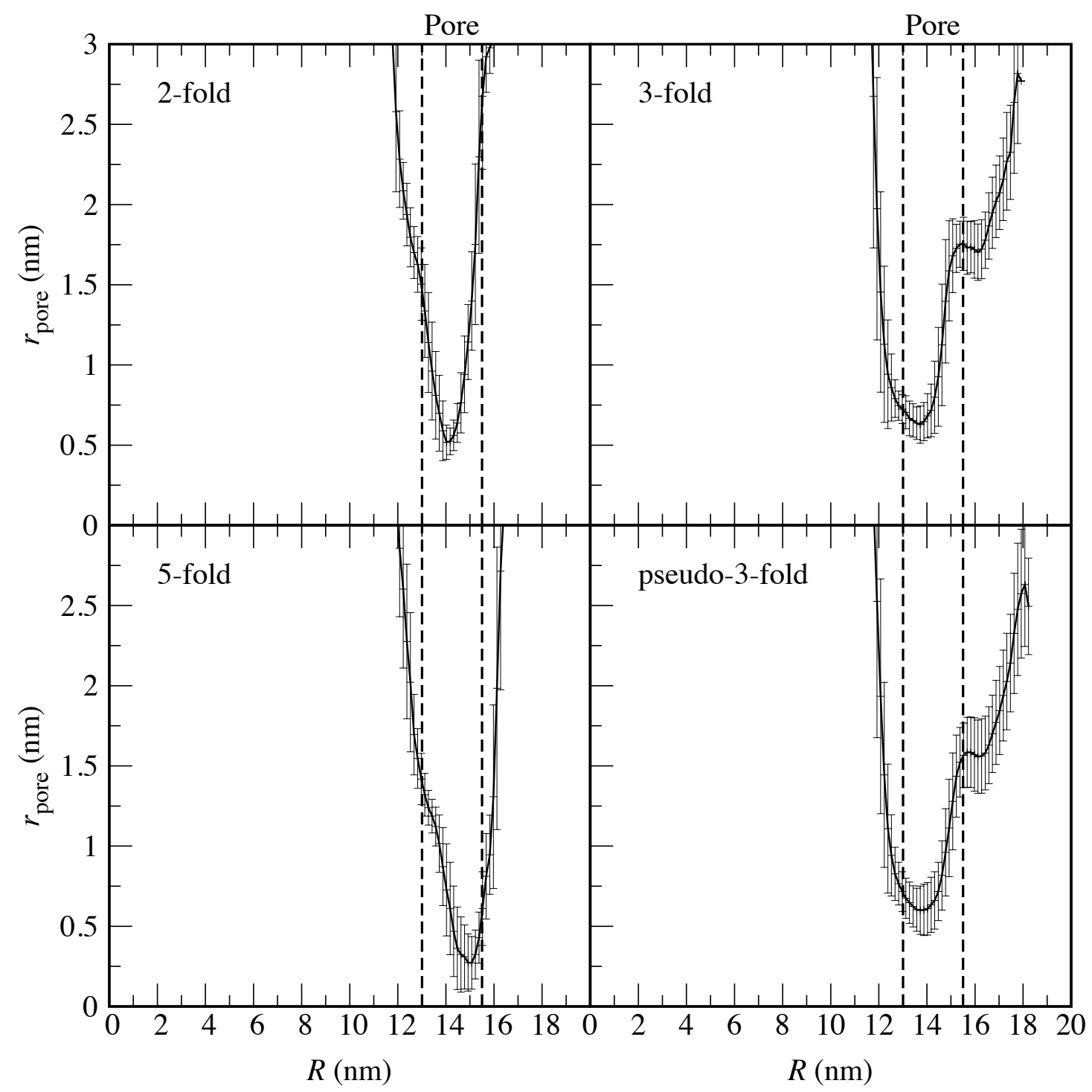

FIG. 11. Averaged pore size $r_{\text {pore }}(R)$ along the rotational symmetry axes. There are 30 pores, 20 pores, 60 pores, and 12 pores along the 2-fold, 3-fold, pseudo-3-fold, and 5-fold rotational symmetry axes in an HBV capsid, respectively. The average was taken over all pores and over $100 \mathrm{~ns}$ trajectories between $t=100 \mathrm{~ns}$ and $200 \mathrm{~ns}$. The error bars represent the standard deviations calculated using the same statistics. Detailed time slices of the distributions for two pores are shown in the Supporting Information.

The side chains of the capsid proteins protrude inside the pores. In other words, the spaces in the pores are narrow in some places and larger in others. As such, pore radius is a function of position in the pore. The calculated pore size profile, $r_{\text {pore }}(R)$, is shown in Fig. 11 for the pores around the 2-fold, 3-fold, pseudo-3-fold, and 5-fold rotational symmetry axes. First, the fluctuations (the error bars in the Figure) of $r_{\text {pore }}(R)$ are large for all the pores. This reflects not 
only the difference in $r_{\text {pore }}(R)$ in the same rotational symmetry axis, but also the fact that the size of each pore fluctuates with time. Fig. S3 shows the time variation of the pore size profiles of the two selected pores along each rotational symmetry axis. We can see that the $r_{\text {pore }}(R)$ profiles are somewhat different, even between pores along the same rotational symmetry axis. In particular, the narrowest positions along the pores are different. The time variation of the $r_{\text {pore }}(R)$ profile is shown in Fig. S3. There is no plateau region $r_{\text {pore }}(R)$ profile in Fig. 11, indicating that the pore was not a straight cylinder.

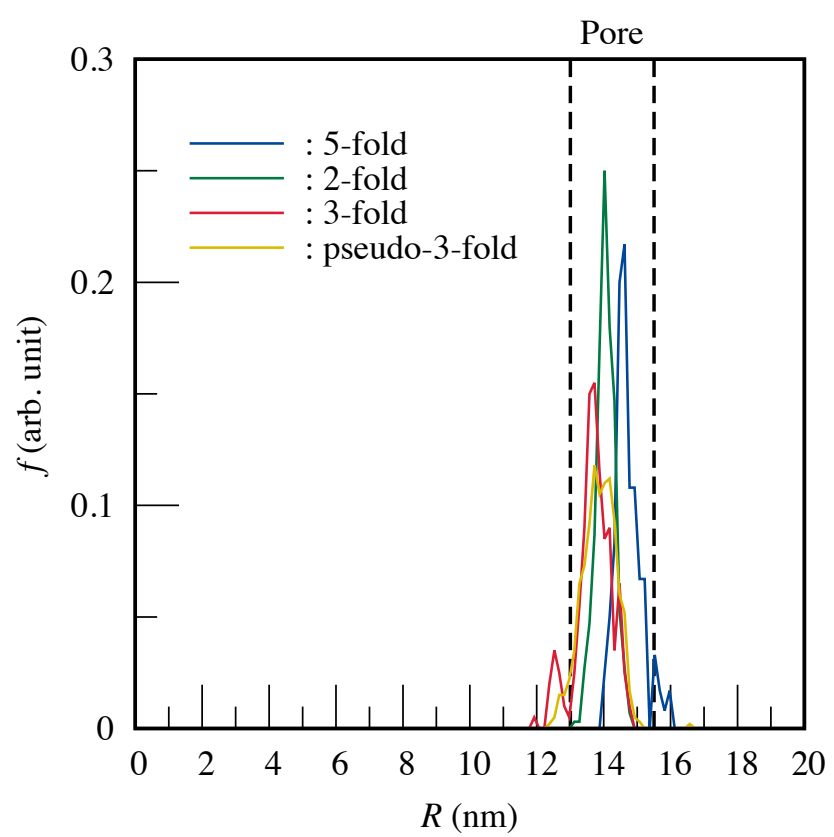

FIG. 12. Distribution of the position of the narrowest space in the pores along the rotational symmetry axes. The distribution was calculated using all structural data from the production run between 100 ns and 200 ns.

From the perspective of substance transportation, the minimum position of the $r_{\text {pore }}(R)$ profile should be related to the rate-determining step in the permeation. The distributions of the minimum positions of the $r_{\text {pore }}(R)$ profiles for the different pores are shown in Fig. 12. As shown in Fig. 12, a peak in the distribution of the minimum positions along the 5-fold rotational symmetry axis was found at the entrance of the pores (at a large $R$ of $14.6 \mathrm{~nm}$ ) for entry into the capsid. The distribution of the pores along the 2-fold rotation symmetry axis exhibited a peak at approximately $R=14.0$. The pores were narrow in the middle. On the other hand, the pores of the 3 -fold rotation symmetry axis were narrowest at $R=13.7 \mathrm{~nm}$, near the exit. The pores of the pseudo-3-fold rotation symmetry axis were also found to be narrow, in the range $R=13.7-14.2 \mathrm{~nm}$, from the middle to the exit of the pores. 


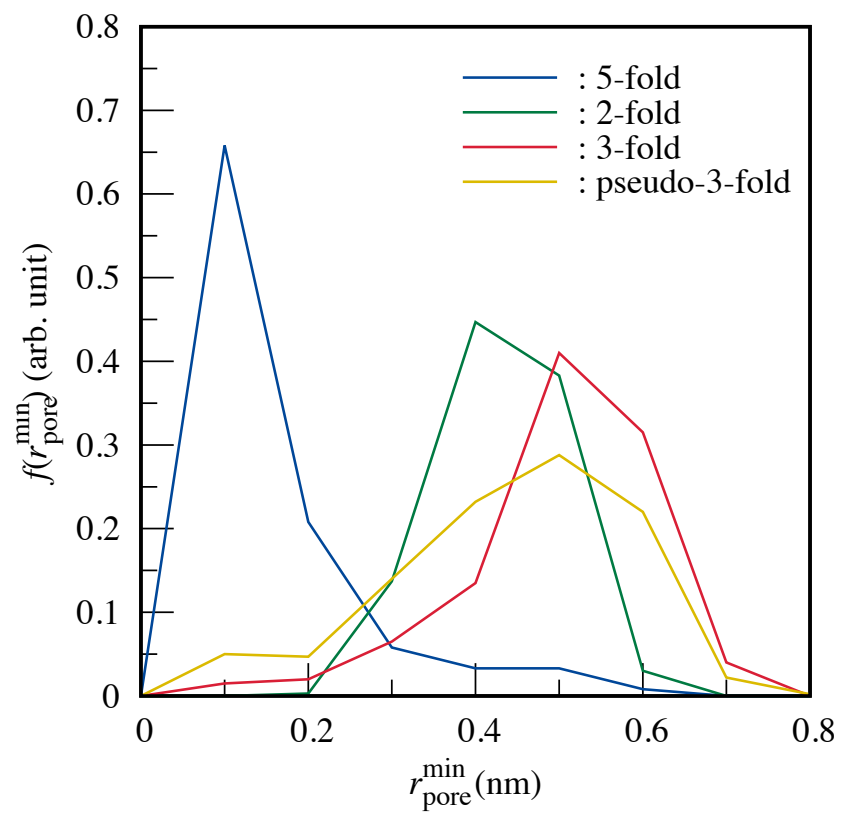

FIG. 13. Distribution of the narrowest space size $\left(r_{\mathrm{pore}}^{\mathrm{min}}\right)$.

Fig. 13 presents the distribution of the narrowest pore radius $\left(r_{\text {pore }}^{\min }\right)$ in the pores. This indicates that the pores have various minimum radii. Furthermore, they were closed at times and open at others. For the 5-fold rotational symmetry axis, the peak was found at approximately $r_{\text {pore }}{ }^{\min }=0.1 \mathrm{~nm}$, indicating that the pores were essentially closed. In contrast, for the 3 -fold and pseudo-3-fold rotational symmetry axes, the pores had a peak at approximately $0.5 \mathrm{~nm}$, suggesting that the pores always maintain a large radius. For the 2-fold rotational symmetry axes, there was a peak at approximately $0.4 \mathrm{~nm}$. Although this was slightly smaller than that of the pores near the 3-fold and pseudo-3-fold rotational symmetry axes, there was clearly a hole. The average sizes of the pores of the 5-fold, 3-fold, pseudo-3-fold, and 2-fold rotational symmetry axes were 0.16 , $0.50,0.44$, and $0.43 \mathrm{~nm}$, respectively. It should be noted that $r_{\text {pore }}{ }^{\min }$ is defined as the distance from the pore center to the atomic center. However, to investigate the pore size from the viewpoint of substance transport, the van der Waals (vdW) radius of the atom must be considered. Assuming a vdW radius of the atom of $0.1 \mathrm{~nm}$, the size of the pore along the 5-fold, 3-fold, pseudo-3-fold, and 2-fold rotational symmetry axes was $0.06 \mathrm{~nm}, 0.40 \mathrm{~nm}, 0.34 \mathrm{~nm}$, and $0.33 \mathrm{~nm}$, respectively. In other words, the space in the 5-fold rotational symmetry axis was smaller than the sizes of the water molecules and ions. In contrast, the other pores were sufficiently large compared to the water molecules and ions. However, they were still smaller than the sizes of the hydrated water molecules and ions. This point is discussed further below, with a focus on the free energy. 

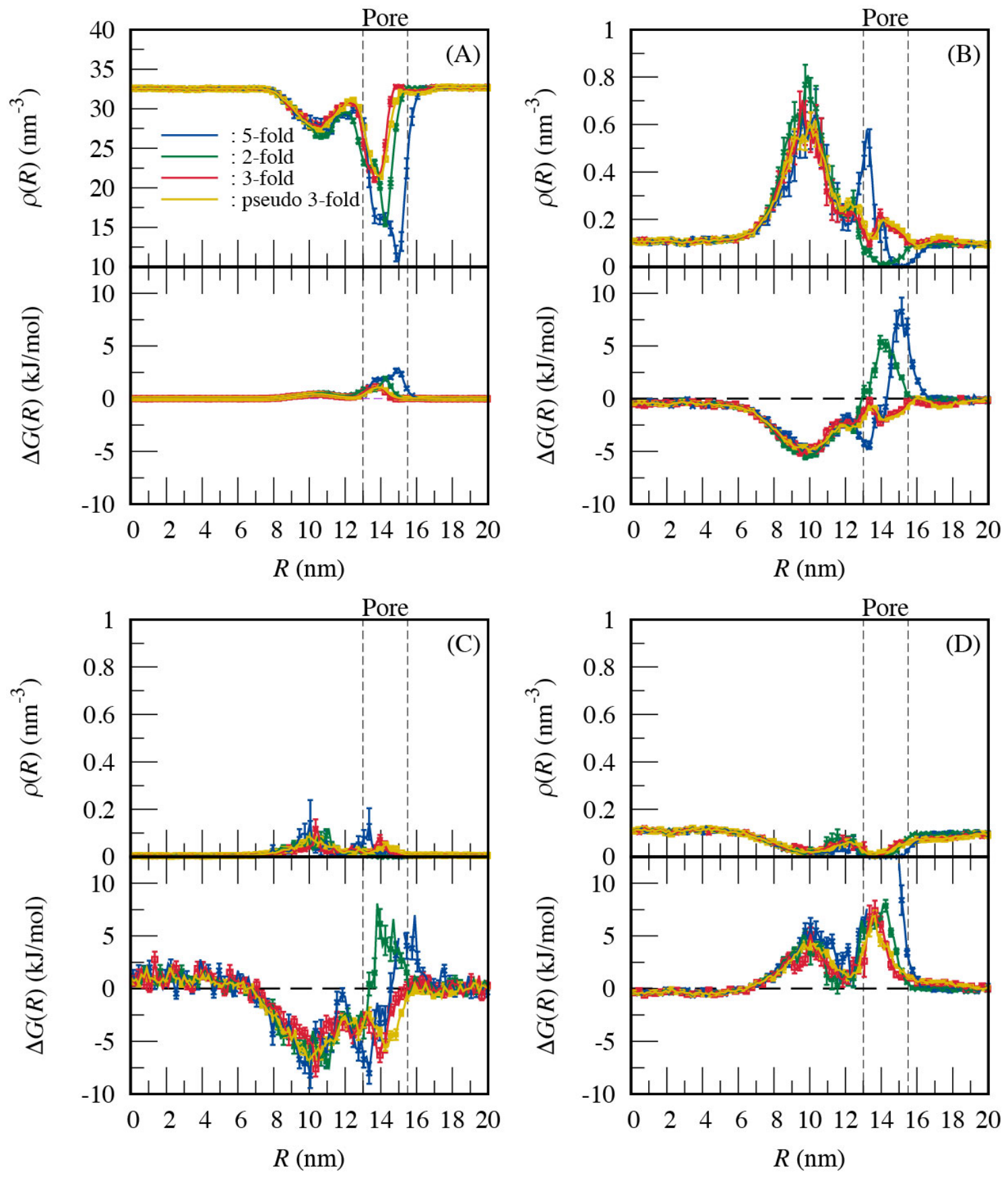

FIG. 14. The calculated density profile and free energy profile of $(A)$ water molecules, (B) potassium ions, $(C)$ sodium ions, and $(D)$ chloride ions as functions of the distance from the center of mass of the HBV. The blue, green, red, and yellow lines indicate the pores along the 5-fold, 2fold, 3-fold, and pseudo-3-fold rotational symmetry axes, respectively.

Fig. 14 presents the calculated density profile, $\rho(r)$, of water molecules, potassium ions, sodium ions, and chloride ions as functions of the distance from the center of mass of the HBV capsid. The free energy profile, calculated using Eq. 1, is also shown. The water molecule has 
free energy barriers of 2.8 and $1.8 \mathrm{~kJ} / \mathrm{mol}$ in the pores along the 5-fold and 2-fold rotational symmetry axes, respectively. The free energy barriers in the pores along the 3 -fold and pseudo3 -fold rotational symmetry axes were both $1.1 \mathrm{~kJ} / \mathrm{mol}$, which was the smallest among the pores. The $\mathrm{vdW}$ radius of a water molecule was approximately $0.14 \mathrm{~nm}$. The estimated pore sizes were $0.06 \mathrm{~nm}, 0.40 \mathrm{~nm}, 0.34 \mathrm{~nm}$, and $0.33 \mathrm{~nm}$ for the 5-fold, 3-fold, pseudo-3-fold, and 2-fold rotational symmetry axes, respectively. The size of the pores was not large enough for water to pass through without reducing the number of hydrogen bonds with the surrounding water. For this reason, an energy barrier was produced in all the pores. The free energy barrier in the pore along the 5-fold rotational symmetry axis was located near the entrance of the pore. The peak in the free energy profile was found in the middle of the pore along the 2-fold rotational symmetry axis. The pores along the 3-fold and pseudo-3-fold rotational symmetry axes were found to have barriers near their exits. Free energy barriers were found at the location of the minimum radius of the pore, as shown in Fig. 12. Because the free energy barrier heights were smaller than the thermal energy $(2.5 \mathrm{~kJ} / \mathrm{mol})$ for all the pores, water molecules were able to easily migrate into the HBV capsid through these pores. Furthermore, although the pores around the 2-fold and 3 -fold rotational symmetry axes were considered hydrophobic and hydrophilic, respectively, the present data demonstrated no significant difference in terms of the free energy barrier for water between these pores.

Next, we investigated the density and free energy profiles of the ions. The radii of $\mathrm{Na}^{+}, \mathrm{K}^{+}$, and $\mathrm{Cl}^{-}$in the crystal structure were $0.10,0.13$, and $0.18 \mathrm{~nm}$, respectively. However, the radii of the hydrated ions were estimated to be $0.38 \mathrm{~nm}, 0.41 \mathrm{~nm}$, and $0.46 \mathrm{~nm}$, respectively. Thus, the ions can not pass through the pores without dehydration. This resulted in the formation of free energy barriers. First, in terms of the cations $\mathrm{Na}^{+}$and $\mathrm{K}^{+}$, a free energy barrier was formed in the pores along the 5-fold and 2-fold rotational symmetry axes. The positions of these barriers corresponded well to the location of the narrowest portions of the pores. Furthermore, the free energy was lower at $r=13 \mathrm{~nm}$ for the pores along the 5-fold rotational symmetry axis. This was due to the negatively charged amino acid residues (RESID: GLU8, GLU117, GLU113, and GLU145). In contrast, hydrophobic residues, PROs, in the pores along the 2-fold rotational symmetry axis produced a free energy barrier for $\mathrm{Na}^{+}$and $\mathrm{K}^{+}$because the cations needed to be dehydrated there. The free energies of $\mathrm{K}^{+}$and $\mathrm{Na}^{+}$were lower inside the pores along the 3 -fold and pseudo-3-fold rotational symmetry axes than that in the bulk $(r=20 \mathrm{~nm})$. This was again caused by negatively charged amino acid residues (RESID: ASP2, ASP4, ASP40, GLU14, and GLU43) in the pores. Hadden et al. ${ }^{14}$ reported that many sodium ions were present in this region. This result is in good agreement with our results. There was a very small increase in the free 
energy around $R=13 \mathrm{~nm}$, as a result of the narrow pores. At $6<R<12 \mathrm{~nm}$, the free energy of $\mathrm{Na}^{+}$and $\mathrm{K}^{+}$had a large negative value due to the negatively charged phosphate groups of CTD and pgRNA.

The free energy barrier of $\mathrm{Cl}^{-}$was high in all pores along the rotational symmetry axes. Negatively charged amino acid residues were observed in the pores along the 5-fold, 3-fold, and pseudo-3-fold rotational symmetry axes. In contrast, hydrophobic amino acid residues were mostly found along the pores around the 2-fold rotational symmetry axis. In addition, owing to the pore size, $\mathrm{Cl}^{-}$needed to be dehydrated to pass through the pores. Taken together, these were the causes of the formation of the significant free energy barrier. In our previous study, ${ }^{15}$ we calculated the free energy profile of ETVTP along the pores around the 2-fold and 3-fold rotational symmetry axes. ETVTP is a nucleotide analog with a charge of $4 \mathrm{e}^{-}$. Although it contains a free energy barrier within the pore, a free energy minimum was found at the entrance of the pore along the 3-fold rotational symmetry axis. The free energy minimum was caused by a structural change in the positively charged N-terminal of the capsid (RESID: MET1). This is a sort of induced fit by the capsid. The capsid may also have structural flexibility to capture nucleotides for reverse transcription. At $6<R<12 \mathrm{~nm}$, the free energy had a large positive value because of the negatively charged phosphate groups of CTD and pgRNA.

The free energies of $\mathrm{K}^{+}$and $\mathrm{Cl}^{-}$were slightly but significantly more negative inside the capsid than outside it by $\sim 0.5 \mathrm{~kJ} / \mathrm{mol}$. This indicates that the ions are stable, irrespective of their signs, when they are located inside the capsid.

We obtained an approximate estimation of the permeation rate across the capsid shell with the aid of primitive transition state theory. The reaction rate constant, $k$, was proportional to the exponential of the activation free energy barrier height $\Delta G^{\ddagger}$ :

$$
k \propto \exp \left(\frac{-\Delta G^{\ddagger}}{R T}\right)
$$

where $T$ and $R$ are the system temperature and gas constant, respectively. We used the calculated free energy barriers for $\Delta G^{\ddagger}$. The ratio of $k$ for each type of ion to that of water is presented in Table III. As shown in Table III, chloride ions were able to permeate through all pores except for 5 -fold at a rate only one-tenth of that of water molecules. The total number of chloride ions that permeated in $1 \mathrm{~ns}$ was approximately 1/3000 that of water (Table II). However, the number of water molecules in the system was 370 times that of the chloride ions. Considering this difference, 
the permeation rate of chloride ions may be estimated to be approximately $1 / 10$ that of water. This is in good agreement with the results presented in Table III.

The calculated $k$ values of the sodium and potassium ions through the pores along the 5 -fold and 2-fold rotational symmetry axes were also 4-7 times smaller than those of the water molecules. In Table II, the total number of potassium and sodium ions that permeated in $1 \mathrm{~ns}$ was approximately $1 / 300$ and $1 / 5000$ that of water, respectively. In addition, the numbers of potassium and sodium ions in the system are approximately $1 / 300$ and $1 / 4000$ that of water molecules, respectively (Table I). Considering the difference in the concentrations, the normalized permeation rates of potassium and sodium ions should be almost the same as that of water. However, this result was not consistent with the calculated $k$ values of the sodium and potassium ions through the pores around the 5-fold and 2-fold rotational symmetry axes, as shown in Table III. Therefore, these ions mainly passed through the pores around the 3-fold and pseudo-3-fold rotational symmetry axes without any free energy penalty.

TABLE III. Ratios of the calculated reaction rate constant of ions to that of water. Since chloride ions did not pass through the pore along the 5 -fold rotational symmetry axis during the $200 \mathrm{~ns}$ MD run, $k$ is not presented. The calculated $k$ values of the sodium and potassium ions through the pores along the 3-fold and pseudo-3-fold rotational symmetry axes are also not shown, because there was no free energy barrier for the permeating ions through the pore along the 3fold and pseudo-3-fold rotational symmetry axes.

\begin{tabular}{lcccc}
\hline & Water & $\mathbf{K}^{+}$ & $\mathbf{N a}^{+}$ & $\mathbf{C l}^{-}$ \\
\hline 5-fold & 1 & 0.14 & 0.14 & - \\
2-fold & 1 & 0.25 & 0.25 & $\mathbf{0 . 0 9}$ \\
3-fold & 1 & - & - & $\mathbf{0 . 0 9}$ \\
pseudo-3-fold & $\mathbf{1}$ & - & - & $\mathbf{0 . 1 1}$ \\
\hline
\end{tabular}

\section{E. Local pressure}

In a previous study, ${ }^{13}$ we calculated the local pressure inside an empty poliovirus capsid. Surprisingly, the results were negative. In general, the pressure in a confined space smaller than the bubble nucleation size can be negative. This is known as a metastable state. The negative pressure found inside the capsid suggested that the capsid was organized in such a way that it can spontaneously contain RNA. In terms of the mechanism, when poliovirus contains RNA inside its capsid, the pressure becomes positive due to Van't Hoff osmotic pressure. Subsequently, the 
total system is stabilized. The local pressure of HBV with pgRNA is shown in Fig. 15. We set the bulk pressure at $r=21.5 \mathrm{~nm}$ to be $0 \mathrm{MPa}$ as a reference. It was clear that the pressure inside the capsid was positive, indicating that the virus with RNA was more stable than the empty capsid, through a process known as physicochemical stabilization.

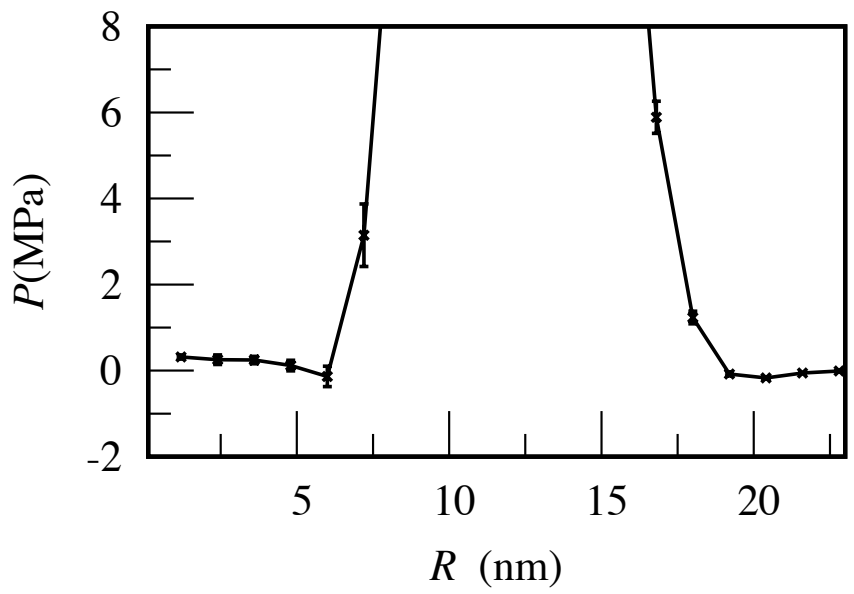

FIG. 15. The calculated local pressure as a function of distance from the center of mass of the HBV capsid. The bulk pressure at $R=21.5 \mathrm{~nm}$ was set to $0 \mathrm{MPa}$.

\section{CONCLUSIONS}

To clarify the microscopic physico-chemical properties of HBV (with pgRNA) in solution, a 200-ns MD calculation was performed. The system reached thermal equilibrium within $100 \mathrm{~ns}$. The calculated RMSF was in good agreement with the experimental results. The structural fluctuations observed in the terminal and spike regions were large, although those in the other regions were small. The calculated radial electric density distribution was in agreement with the experimental results, although the calculated radial electric density distribution reflected a detailed structure that was not observed in the experiment with large errors.

Next, the electric field around the HBV capsid was calculated. The electric field yielded only a very small force on negatively charged substances, such as nucleotides, at distances greater than $20 \mathrm{~nm}$. In other words, negatively charged substances did not recognize HBV. At distances less than $20 \mathrm{~nm}$, repulsive forces on negatively charged particles were detected only around spikes, although no force was detected in the other regions. Thus, negatively charged particles were able to approach the capsid by random walk trajectories while avoiding spikes. Weak attractive average forces acted on negative charges just above the floor of the capsid. Forces were produced by charged residues around the 5-fold rotational symmetry axis. However, the forces were weaker 
than the thermal forces, allowing negatively charged substances to migrate over the floor to search for pores.

We also calculated the permeation rates of water and ions through the pores in the HBV capsid. The permeation rates of the potassium and sodium ions were 71 and $60 \mathrm{~ns}^{-1} \cdot\left(\mathrm{mol} / \mathrm{dm}^{3}\right)^{-1}$, respectively, while that of chloride ion was as low as $8.7 \mathrm{~ns}^{-1} \cdot\left(\mathrm{mol} / \mathrm{dm}^{3}\right)^{-1}$. Thus, the calculated exchange rate of chloride ions between the inside and outside of the capsid was eight times slower than that of potassium and sodium ions. This result shows that HBV exhibits ion selectivity when it captures the ions inside it.

The free energy profiles were calculated for water molecules and ions as functions of the distance from the center of mass of the capsid along each pore. Free energy barriers for water molecules were observed in all pores, although they were smaller than the thermal energy. Thus, water molecules were able to enter and exit any pores of the HBV capsid. On the other hand, potassium and sodium ions had no free energy barriers in the pores along the 3 -fold and pseudo3 -fold rotational symmetry axes. Therefore, positively charged ions migrated into and out of the pores around the 3-fold and pseudo-3-fold rotational symmetry axes. In contrast, free energy barriers of more than approximately $8 \mathrm{~kJ} \cdot \mathrm{mol}^{-1}$ for chloride ions were observed in all the pores. This indicates that chloride ions were able to permeate to a degree by crossing the barrier. Furthermore, the difference in free energy between the inside and outside of the capsid was -0.5 $\mathrm{kJ} / \mathrm{mol}$ for both potassium and chloride ions. The ions were more stable within the HBV capsid, irrespective of their sign of charge. The HBV capsid can provide a stable environment for charged particles, such as nucleotides, supporting their capture for reverse transcription.

\section{SUPPLEMENTARY MATERIAL}

See supplementary material for detailed HBV modeling methods. In addition, the supporting figure referred in the text is also provided in the supplementary material.

\section{ACKNOWLEDGMENTS}

We would like to thank Prof. Adam Zlotnic for his helpful discussions. We also thank Dr. Hajime Imai for his collaboration during the early stages of this project and Mr. Shuhei Tsurumaki for his help with data processing. This work was supported by MEXT as "Priority Issue on PostK computer" (Building Innovative Drug Discovery Infrastructure Through Functional Control of 
Biomolecular Systems) (project IDs: hp150269, hp160223, hp170255, hp180191, and hp190171) and "General Issue on Post-K computer" (Absorption Mechanism of Inhibitor into Capsid by using All-Atom Molecular Dynamics of Hepatitis B Virus in Solution) (project IDs: hp160143, hp170205, hp180131, and hp190129) using computational resources of the K computer provided by the RIKEN Center for Computational Science through the HPCI System Research project. This work was also partially supported by MEXT as the "Program for Promoting Research on the Supercomputer Fugaku" (Biomolecular dynamics and function in a living cell using atomistic and coarse-grained simulations) (project IDs: hp200135 and hp210177). Calculations were also performed by supercomputers at the Research Center for Computational Science, Okazaki, Japan, at the Information Technology Center of Nagoya University, at the Information Initiative Center, Hokkaido University, at the Cybermedia Center, Osaka University, and at the Institute for Solid State Physics, the University of Tokyo.

\section{DATA AVAILABILITY}

The data that supports the findings of this study are available within the article and its supplementary material.

\section{REFERENCES}

${ }^{1}$ WHO, Global Hepatitis Report 2017.

${ }^{2}$ J. C. -Y. Wang, D. G. Nickens, T. B. Lentz, D. D. Loeb, and A. Zlotnick, Proc. Natl. Acad. Sci.

U. S. A. 111/31, 11329-11334 (2014) [DOI: 10.1073/pnas.1321424111].

${ }^{3}$ J. C. Wang, M. S. Dhason, and A. Zlotnick, PLOS Pathog. 8/9, e1002919 (2012) [DOI: 10.1371/journal.ppat.1002919].

${ }^{4}$ A. Zlotnick, B. Venkatakrishnan, Z. Tan, E. Lewellyn, W. Turner, and S. Francis, Antiviral Res. 121, 82-93 (2015) [DOI: 10.1016/j.antiviral.2015.06.020].

${ }^{5}$ X. Yu, L. Jin, J. Jih, C. Shih, and Z. H. Zhou, PLOS ONE 8/9, e69729 (2013) [DOI: 10.1371/journal.pone.0069729].

${ }^{6}$ K. A. Dryden, S. F. Wieland, C. Whitten-Bauer, J. L. Gerin, F. V. Chisari, and M. Yeager, Mol. Cell 22/6, 843-850 (2006) [DOI: 10.1016/j.molcel.2006.04.025].

${ }^{7}$ A. Zlotnick, N. Cheng, J. F. Conway, F. P. Booy, A. C. Steven, S. J. Stahl, and P. T. Wingfield, Biochemistry 35/23, 7412-7421 (1996) [DOI: 10.1021/bi9604800] [8652518]. 
${ }^{8}$ C. Uetrecht, C. Versluis, N. R. Watts, W. H. Roos, G. J. L. Wuite, P. T. Wingfield, A. C. Steven, and A. J. R. Heck, Proc. Natl. Acad. Sci. U. S. A. 105/27, 9216-9220 (2008) [DOI: 10.1073/pnas.0800406105].

${ }^{9}$ S. A. Wynne, R. A. Crowther, and A. G. W. Leslie, Mol. Cell 3/6, 771-780 (1999) [DOI: 10.1016/s1097-2765(01)80009-5].

${ }^{10}$ P. L. Freddolino, A. S. Arkhipov, S. B. Larson, A. McPherson, and K. Schulten, Structure 14/3, 437-449 (2006) [DOI: 10.1016/j.str.2005.11.014].

${ }^{11}$ D. S. Larsson, L. Liljas, and D. van der Spoel, PLOS Comput. Biol. 8/5, e1002502 (2012) [DOI: 10.1371/journal.pcbi.1002502].

${ }^{12}$ E. Tarasova, V. Farafonov, R. Khayat, N. Okimoto, T. S. Komatsu, M. Taiji, and D. Nerukh, J. Chem. Phys. Lett., 8, 779 (2017).

${ }^{13}$ Y. Andoh, N. Yoshii, A. Yamada, K. Fujimoto, H. Kojima, K. Mizutani, A. Nakagawa, A. Nomoto, and S. Okazaki, J. Chem. Phys. 141/16, 165101 (2014) [DOI: 10.1063/1.4897557].

${ }^{14}$ J. A. Hadden, J. R. Perilla, C. J. Schlicksup, B. Venkatakrishnan, A. Zlotnick, and K. Schulten, eLife 7, e32478 (2018) [DOI: 10.7554/eLife.32478].

${ }^{15}$ K. Fujimoto, M. Fukai, R. Urano, W. Shinoda, T. Ishikawa, K. Omagari, Y. Tanaka, A. Nakagawa, and S. Okazaki, Pure Appl. Chem. 92/10, 1585-1594 (2020) [DOI: 10.1515/pac2020-0109].

${ }^{16}$ G. Zhao, J. R. Perilla, E. L. Yufenyuy, X. Meng, B. Chen, J. Ning, J. Ahn, A. M. Gronenborn, K. Schulten, C. Aiken, P. Zhang, Nature 497/7451, 643-646 (2013) [DOI: 10.1038/nature12162]. ${ }^{17}$ J. R. Perilla, K. Schulten, Nat. Commun. 8, 15959 (2017) [DOI: 10.1038/ncomms15959].

${ }^{18}$ F. Pastor, C. Herrscher, R. Patient, S. Eymieux, A. Moreau, J. Burlaud-Gaillard, F. Seigneuret, H. de Rocquigny, P. Roingeard, and C. Hourioux, Sci. Rep. 9/1, 16178 (2019) [DOI: 10.1038/s41598-019-52824-z].

${ }^{19}$ Q. Zhao, Z. Hu, J. Cheng, S. Wu, Y. Luo, J. Chang, J. Hu, and J. T. Guo, J. Virol. 92/13, e02139 (2018) [DOI: 10.1128/JVI.02139-17].

${ }^{20}$ L. Selzer, R. Kant, J. C. -Y. Wang, B. Bothner, and A. Zlotnick, J. Biol. Chem. 290/47, 2858428593 (2015) [DOI: 10.1074/jbc.M115.678441].

${ }^{21}$ N. Leontis, E. Westhof, and J. M. Bujnicki, RNA, 3D Structure Analysis and Prediction (Springer, 2012).

${ }^{22}$ S. J. Flint, L. W. Enquist, V. R. Racaniello, and A. M. Skalka, Principles of Virology Second Edition (Am. Society for Microbiology, 2003).

${ }^{23}$ J. Gumbart, L. G. Trabuco, E. Schreiner, E. Villa, K. Schulten, Struct. 17/11, 1453-1461 (2009) [DOI: 10.1016/j.str.2009.09.010]. 
${ }^{24}$ W. Humphrey, A. Dalke, and K. Schulten, J. Mol. Graph. 14/1, 33-38 (1996) [DOI: 10.1016/0263-7855(96)00018-5].

${ }^{25}$ M. N. Ucisik, P. C. Bevilacqua, and S. Hammes-Schiffer, Biochemistry 55/27, 3834-3846 (2016) [DOI: 10.1021/acs.biochem.6b00203].

${ }^{26}$ J. C. Bowman, T. K. Lenz, N. V. Hud, and L. D. Williams, Curr. Opin. Struct. Biol. 22/3, 262272 (2012) [DOI: 10.1016/j.sbi.2012.04.006].

${ }^{27}$ B. Alberts, A. Johnson, J. Lewis, D. Morgan, M. Raff, and K. Roberts, Molecular Biology of the Cell 6th edition, (2014).

${ }^{28}$ W. G. Hoover, Phys. Rev. A 31/3, 1695 (1985) [DOI: 10.1103/PhysRevA.31.1695].

${ }^{29}$ H. C. Andersen, J. Chem. Phys. 72/4, 2384-2393 (1980) [DOI: 10.1063/1.439486].

${ }^{30}$ R. B. Best, X. Zhu, J. Shim, P. E. M. Lopes, J. Mittal, M. Feig, and A. D. Mackerell, J. Chem. Theory Comput. 8/9, 3257-3273 (2012) [DOI: 10.1021/ct300400x].

${ }^{31}$ E. J. Denning, U. D. Priyakumar, L. Nilsson, and A. D. Mackerell, J. Comput. Chem. 32/9, 1929-1943 (2011) [DOI: 10.1002/jcc.21777].

${ }^{32}$ D. Beglov and B. Roux, J. Chem. Phys. 100/12, 9050-9063 (1994) [DOI: 10.1063/1.466711].

${ }^{33}$ A. D. Mackerell, Jr., D. Bashford, M. Bellott, R. L. Dunbrack, Jr., J. D. Evanseck, M. J. Field, S. Fischer, J. Gao, H. Guo, S. Ha, D. Joseph-McCarthy, L. Kuchnir, K. Kuczera, F. T. K. Lau, C. Mattos, S. Michnick, T. Ngo, D. T. Nguyen, B. Prodhom, W. E. Reiher, B. Roux, M. Schlenkrich, J. C. Smith, R. Stote, J. Straub, M. Watanabe, J. Wiórkiewicz-Kuczera, D. Yin, and M. Karplus. Jr., J. Phys. Chem. B 102/18, 3586-3616 (1998) [DOI: 10.1021/jp973084f]

${ }^{34}$ Y. Andoh, N. Yoshii, K. Fujimoto, K. Mizutani, H. Kojima, A. Yamada, S. Okazaki, K. Kawaguchi, H. Nagao, K. Iwahashi, F. Mizutani, K. Minami, S. Ichikawa, H. Komatsu, S. Ishizuki, Y. Takeda, and M. Fukushima, J. Chem. Theory Comput. 9/7, 3201-3209 (2013) [DOI: 10.1021/ct400203a].

${ }^{35}$ J. F. Greengard, The Rapid Evaluation of Potential Fields in Particle Systems (MIT Press, Cambridge, 1988).

${ }^{36}$ G. J. Martyna, M. E. Tuckerman, D. J. Tobias, and M. L. Klein, Mol. Phys. 87/5, 1117-1157 (1996) [DOI: 10.1080/00268979600100761].

${ }^{37}$ G. J. Martyna, D. J. Tobias, and M. L. Klein, J. Chem. Phys. 101/5, 4177-4189 (1994) [DOI: 10.1063/1.467468].

${ }^{38}$ A. Yonezawa, T. Watanabe, M. Yokokawa, M. Sato, and K. Hirao, in Proceedings of International Conference for High Performance Computing, Networking, Storage, and Analysis (Association for Computing Machinery (ACM), Washington, DC, 2011), pp. 1-8. 
${ }^{39}$ K. N. Trueblood, H. B. Bürgi, H. Burzlaff, J. D. Dunitz, C. M. Gramaccioli, H. H. Schulz, U. Shmueli, and S. C. Abrahams, Acta Crystallogr. A Found. Crystallogr. 52/5, 770-781 (1996) [DOI: 10.1107/S0108767396005697].

${ }^{40}$ R. Bartenschlager and H. Schaller, E.M.B.O. J. 11/9, 3413-3420 (1992) [DOI: 10.1002/j.14602075.1992.tb05420.x].

${ }^{41}$ N. R. Watts, J. F. Conway, N. Cheng, S. J. Stahl, D. M. Belnap, A. C. Steven, and P. T. Wingfield, E.M.B.O. J. 21/5, 876-884 (2002) [DOI: 10.1093/emboj/21.5.876].

${ }^{42}$ V. Bruss, World J. Gastroenterol. 13/1, 65-73 (2007) [DOI: 10.3748/wjg.v13.i1.65].

${ }^{43}$ J. Kim and J. Wu, Biophys. J. 107/6, 1453-1461 (2014) [DOI: 10.1016/j.bpj.2014.07.032].

${ }^{44}$ O. S. Smart, J. G. Neduvelil, X. Wang, B. A. Wallace, and M. S. P. Sansom, J. Mol. Graph. 14/6, 354-360 (1996) [DOI: 10.1016/S0263-7855(97)00009-X].

${ }^{45}$ L. Wittkop, A. Schwarz, A. Cassany, S. Grün-Bernhard, M. Delaleau, B. Rabe, C. Cazenave, W. Gerlich, D. Glebe, and M. Kann, Cell. Microbiol. 12/7, 962-975 (2010) [DOI: 10.1111/j.14625822.2010.01444.x].

This article has been submitted to The Journal of Chemical Physics. 